\title{
Cleaning and characterization of objects of cultural value by laser ablation
}

\author{
Gabriel M. Bilmes $^{* a}$, César Freisztav ${ }^{a}$, Daniel Schinca ${ }^{a}$, Alberto Orsetti ${ }^{b}$ \\ ${ }^{a}$ Centro de Investigaciones Opticas (CONICET-CIC) and Universidad Nacional de La Plata, \\ La Plata, Argentina. \\ ${ }^{\mathrm{b}}$ Area de Conservación y Restauración, Secretaría de Cultura, Gobierno de la Ciudad de Bs.As. \\ Buenos Aires, Argentina.
}

\begin{abstract}
Surface ablation with nanosecond laser pulses was applied to preservation, cleaning and compositional identification of objects of cultural value. On one hand, treatments of fabrics, coins, bones, and other archeological objects are shown, as well as applications to the preservation of covers, front of books and old manuscripts made in rag paper. Damage fluence thresholds for 17 different XIX ${ }^{\text {th }}$ century types of papers, made by processing textiles, were determined. On the other hand, we use the spectroscopic analysis of the plasma generated as a result of laser ablation (LIBS- laser Induced Breakdown Spectroscopy-) for the determination of the elementary composition of unique pieces in anthropology and archaeology. In particular, we show applications to the identification of trace elements in Hominide teeth, of interest concerning the analysis of eating habits. We also apply LIBS to the determination of the composition of acheological objects belonging to different pre-Columbian cultures.
\end{abstract}

Keywords: Laser ablation; Laser cleaning, LIBS, Art conservation/restoration

\section{INTRODUCTION}

During the last 20 years optical methods and particularly laser processing of materials and related techniques were increasingly applied to the preservation, cleaning and characterization of objects of cultural value ${ }^{1-6}$. The development of appropiate laser systems and new instruments, together with a better knowledge of the mechanisms involved during the interaction of laser with surfaces, boosted these methods into competitive options respect to traditional ones.

Laser action on materials is strongly dependent on the different parameters involved (i.e. pulse duration, fluence, excitation wavelength, and properties of the surface material). Laser pulses of short duration (below a few ns) and sufficient fluence (energy per unit area) can produce surface ablation. ${ }^{6}$, characterized by material extraction, plasma formation (with light emission) and sound emission. It was previously demonstrated ${ }^{7,8}$ that sound waves produced during ablation procesess can be used for real time measurement of surface cleanliness, avoiding most of the drawbacks of the traditional methods. The basis of this procedure relies on the fact that the intensity of the sound is proportional to the amount of dirt on the surface, and provides a direct measurement of its cleanliness 9 .

Material extraction during laser ablation can be used for surface cleaning. In this case the light emitted by the laser is absorbed by the layer of surface dirt, typically darker than the substrate. In most cases surface dirt can be described as a thin film of organic substances, as oil or grease, in which a more or less homogeneous distribution of particles of different compounds is present. Most of these particles are black, giving the dirt its dark aspect.

Depending on the material, ablation is produced above certain threshold fluence. In cleaning applications the excitation fluence must be appropriate to eliminate the surface dirt without affecting the substrate. Then the excitation fluence must be enough to produce dirt ablation, but it should be below the threshold for substrate ablation. Under this conditions once the surface is cleaned, additional laser shots do not produce any effect.

* gabrielb@ciop.unlp.edu.ar. Casilla de Correo 124, (1900) TE-FAX: 55-221-4712771

Optical Methods for Arts and Archaeology, edited by Renzo Salimbeni, Luca Pezzati, Proceedings of SPIE Vol. 5857 (SPIE, Bellingham, WA, 2005) $\cdot 0277-786$ X/05/\$15 $\cdot$ doi: $10.1117 / 12.612671$

Proc. of SPIE 585704-1 
The spectral analysis of the plasma generated after laser ablation allows the possibility of determination of material composition. In the last years Laser Induced Breakdown Spectroscopy (LIBS) emerged as one of the most versatile and powerful methods for determination of elementary composition of unique pieces of cultural value $e^{6,10,11}$

In a previous work ${ }^{12}$ we show that, modyfing the laser fluence, ablation of surface dirt or substrate material can be performed independently. This procedure can be made at real time simultaneously with laser cleaning. On the other hand, using higher fluences, characterization of substrate composition of unique pieces can be performed.

In this work we show examples of application of laser ablation with nanosecond laser pulses for preservation, cleaning and identification of the composition of objects of cultural value.

\section{EXPERIMENTAL}

A Q-switched Nd: YAG laser with pulse duration of ca. $7 \mathrm{~ns}$ (FWHM) operating at different wavelengths $\lambda=$ $1064 \mathrm{~nm}, \lambda=532 \mathrm{~nm}$ and $\lambda=355$ was used working at repetition rates of up to $10 \mathrm{~Hz}$. Excitation fluence was changed by focusing with apropiate lens and by using a neutral density wedge filter to change the energy of the laser pulse. Laser fluences $(\mathrm{F})$ ranging from $0<\mathrm{F}<4.5 \mathrm{~J} / \mathrm{cm}^{2}$ were used. Energy was controlled by splitting part of the laser beam to a pyroelectric detector. In order to change the impinging position of the beam, the sample was mounted on a xyz controlled positioning step motor system.

For the LIBS experiments, the spectrum of the plasma was acquired focusing the light by means of a lens to a multimode fiber optic attached to a cross dispersion Echelle spectrometer (Mechelle Multichannel Instruments) with a CCD incorporated camera. Data was sent to a computer and processed with appropriate software that gives the whole spectra covering the 200 to $1100 \mathrm{~nm}$ region with a $0.3 \mathrm{~nm}$ spectral resolution. This combination enables simultaneous detection of a large spectral range in a single laser shot. To maximize the signal to background ratio we use a spatial screening procedure that allow to collect only light emitted by regions of the plasma far from the surface ${ }^{12}$.

\section{RESULTS AND DISCUSSION}

\subsection{Laser cleaning of objects}

Figure 1 shows a fragment of fabric (cáñamo), of the XIX century, founded in an urban excavation of one of the older houses of the city of Buenos Aires. This fragment was buried and most of the dirt is stacked dust on grease. The traditional methods for cleaning are brushing and vacuum cleaners with suction regulators, but in cases like this one they may destroy the sample. On the contrary, laser cleaning avoids mechanical contact and undesired manipulation of the sample.

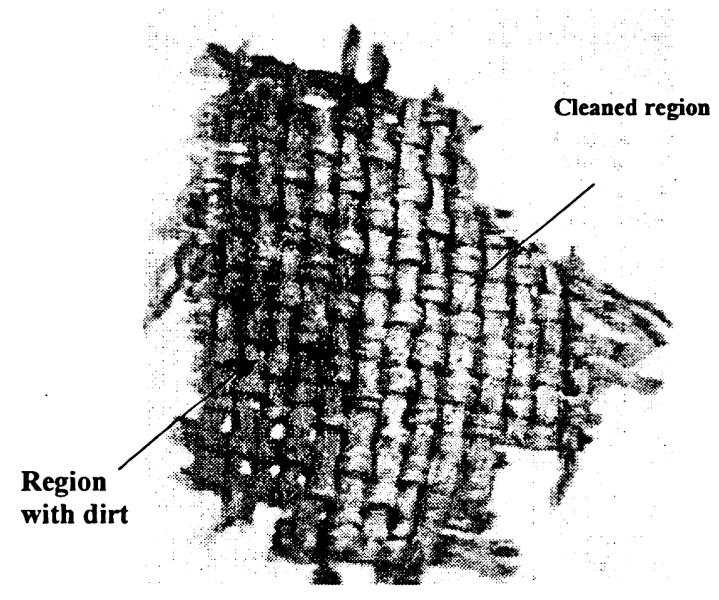

Figure 1. Laser cleaning of fabric of $\mathrm{XIX}^{\text {th }}$ century. Origin: urban archeology of the city of Buenos Aires 
Figure 2 is an example of laser cleaning of bones. In this case we show the inside face of a calota belonging to a precolumbian craneous. The dirt is mainly composed by particles, grease and stacked earth. Usually the traditional cleaning method is based on neutral detergent wash under controlled humidity, or application of alcohols or other solvents that sometimes affect the material. Laser cleaning is dry, and regions with rough surfaces and weakened zones can be easily treated.

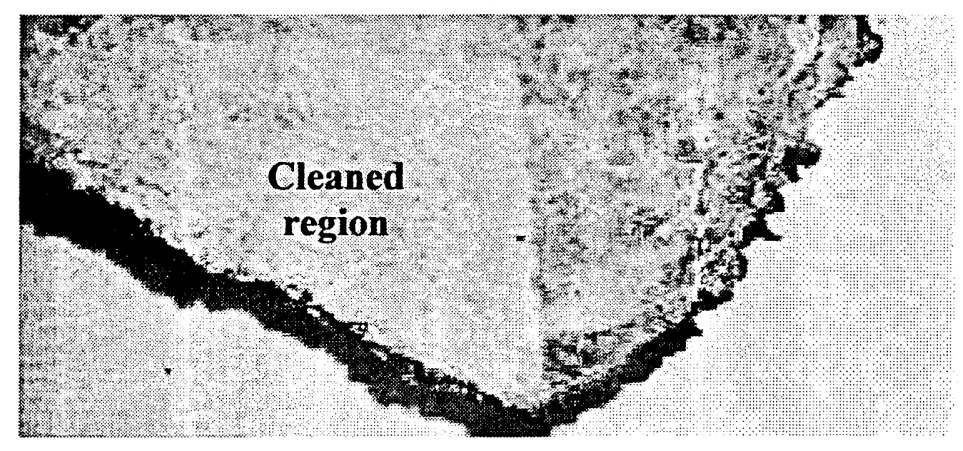

Figure 2. Laser cleaning of a calota of a Precolumbian craneous

Figure 3 shows results of laser cleaning of a coin of 1891. Surface dirt in this case is grease with black particles belonging to manipulation, and oxides. Usually the conventional cleaning is by means of electrolytic or electrochemical baths or acids and alkaline solvents. In some cases these treatments produce erosion of the material, thus altering the shape of the imprinted image.
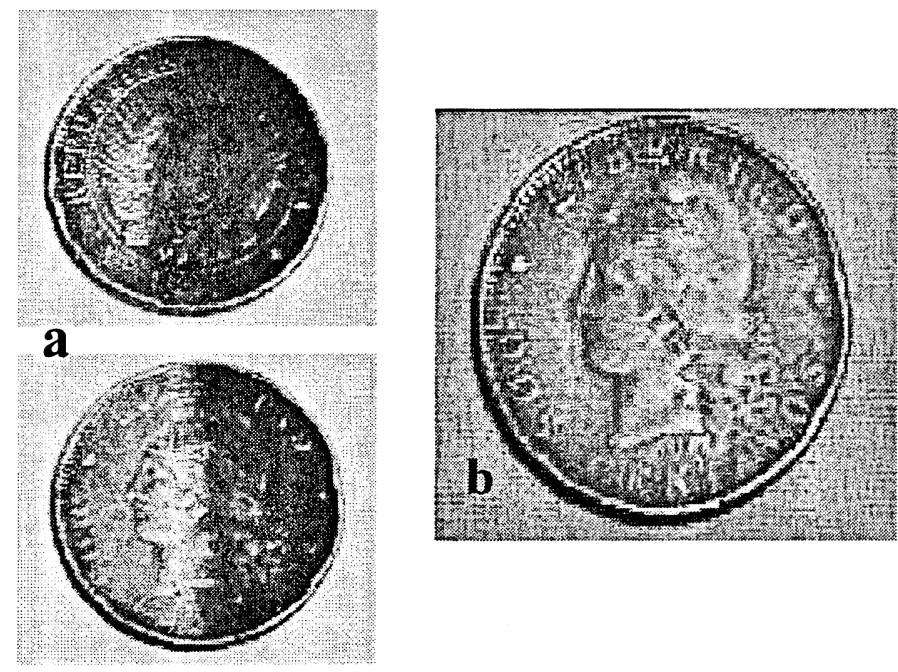

Figure 3: Laser cleaning of a coin of 1891. a) Partial treatment in each face .b) Final state

Laser cleaning of papers and parchments was previously studied showing that treatments require an appropriate knowledge of the type of dirt to be cleaned and the characteristics of the substrate ${ }^{13-15}$.

Typical surface dirt in papers, books, and manuscripts are caused by use and manipulation, that produce a thin film of grease with particles of dust, pigments and sometimes candle soot. This is very frequent to find in covers and fronts of books. The traditional cleaning procedure is by means of dry cleaning with softy rubbers or glass fiber pencils. 
Sometimes water and another solvents can be used. These cleaning procedures produce abrasion and damage of the material causing loss of its mechanical properties.

Figure 4, 5 and 6 show examples of laser cleaning of covers, front and backs of books (clear rectangles).

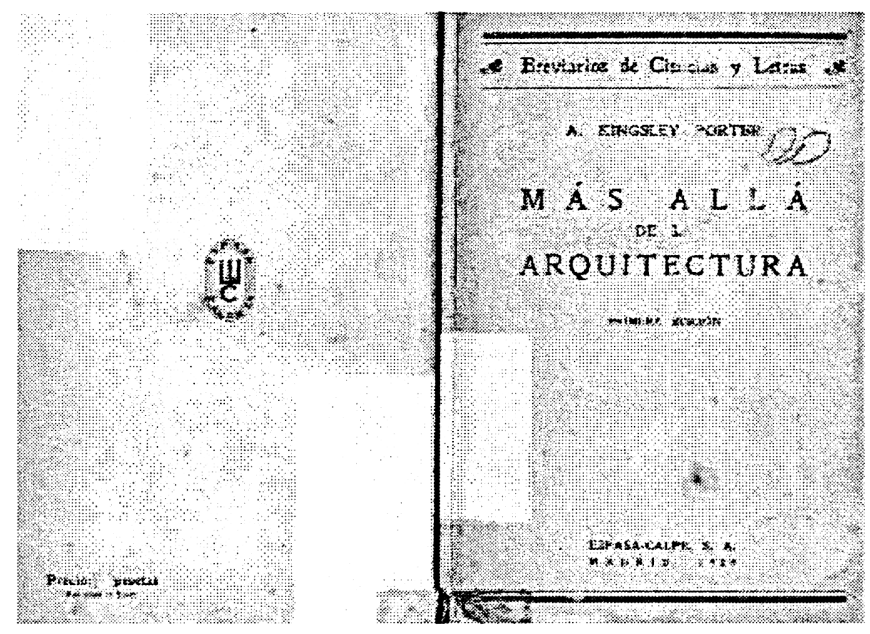

Figure 4. Laser cleaning of cover of books

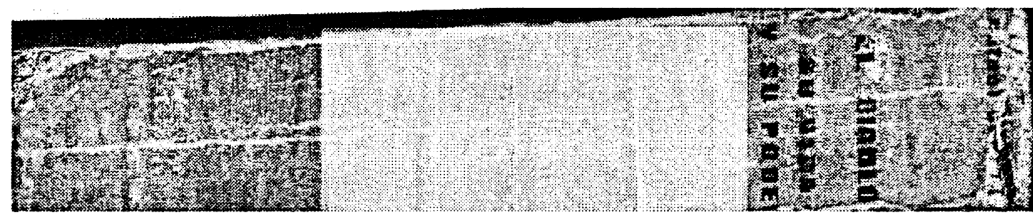

Figure 5. Laser cleaning of a back of a book

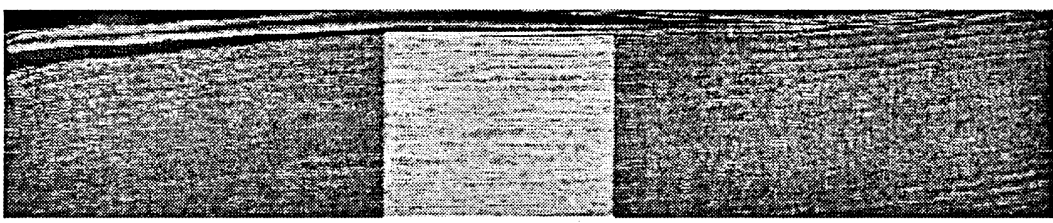

Figure 6. Laser cleaning of edge of a book

Restoration of documents and antique papers usually require cleaning of stains of grease, soot and handling dirt and sometimes ink and pencil writings that must be eliminated. In the case of manuscripts before the $\mathrm{XX}^{\text {th }}$ century most of them were fabricated by processing textiles (Rag paper). For this type of papers, before laser cleaning, we study the threshold damage fluences as a function of wavelength. We selected a collection of 17 different types of papers belonging to the National General Archive of Argentina. Each sample was treated with increasing values of fluence in different regions of the paper. In each case the energy of excitation and the area was measured. Damage was controlled by microscope inspection.

Damage fluence threshold values c.a. $1,2,2$ and $2,8 \mathrm{~J} / \mathrm{cm}^{2}$ were found for excitation wavelengths of 355,532 , and $1064 \mathrm{~nm}$ respectively. Taking into account this result we found that fluences one order of magnitude lower than this values (typically $0,3 \mathrm{~J} / \mathrm{cm}^{2}$ ) are the best suited for laser cleaning of this type of papers, without any damage of the 
substrate. Figure 7 shows examples of laser cleaning of $\mathrm{XIX}^{\text {th }}$ century manuscripts, made on rag paper, written with ferrogalic inks and covered by a dirt film of candle soot.

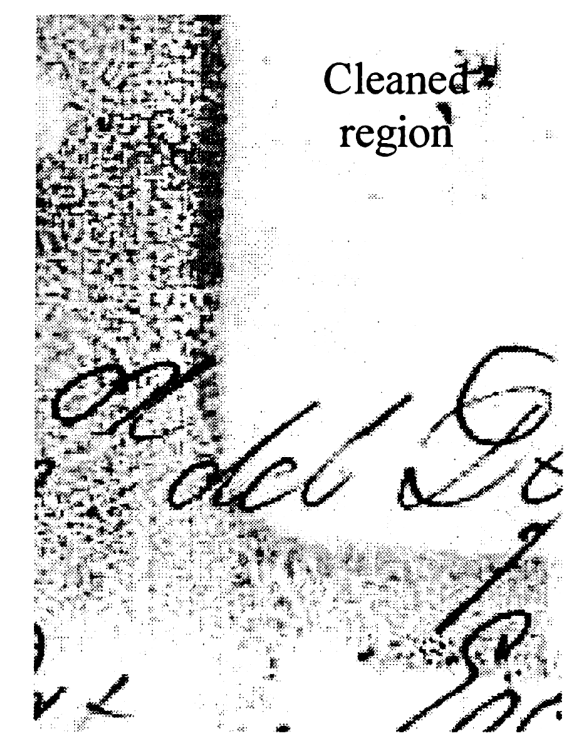

Figure 7: Laser cleaning $(\square=1064 \mathrm{~nm})$ of a manuscript of $\mathrm{XIX}^{\text {th }}$ century made in rag paper
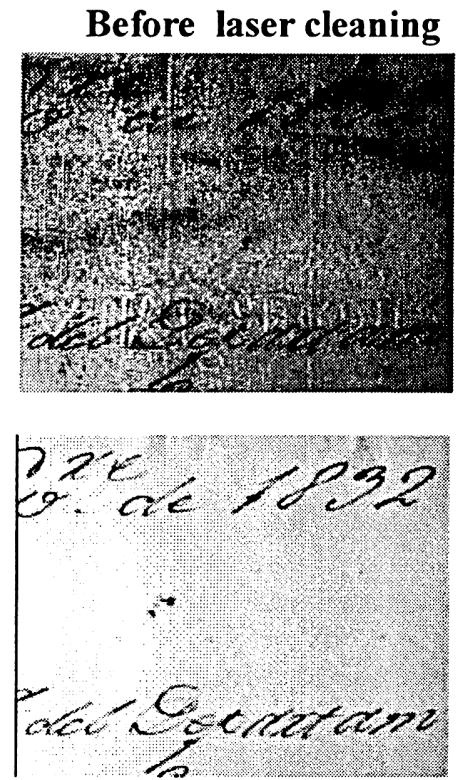

After laser cleaning

We performed also laser cleaning treatments on other materials like leather of seal, belonging to the Argentine zone of Antarctica and used at the beginning o the $\mathrm{XX}$ th century for clothing. In this case the dirt was grease and fungus. In this case laser cleaning allowed the leather to recover its original texture and shine. We also cleaned the inside of glass bottles of the XIX ${ }^{\text {th }}$ century by using a method previously described ${ }^{16}$.

\subsection{Material composition determination}

One of the main data retreving pieces in anthropology are teeth, which yield information about alimentary habits and community life conditions of the studied individuals. Trace elements like $\mathrm{Mg}, \mathrm{Sr}, \mathrm{Ba}, \mathrm{Cu}, \mathrm{Sn}, \mathrm{Va}$ are indicative of different feeding diets. Conventional techniques for trace concentration determination imply partial or total destruction of the pieces which in most cases are unique. We show how LIBS is an option for this type of studies, taking advantage of its microdestructive nature and its oustanding posibility of in situ and real time depth profiling.

As part of an ongoing project ${ }^{17}$ several neolithic, Middle Ages and current teeth from different geographic regions were analized using the LIBS set up already described. The presence of Ca (I y II) ; K I, $\mathrm{Na} \mathrm{I,} \mathrm{Mg}$ I y Sr (I y II) was searched for and identified in the enamel and dentine spectra. Since $\mathrm{Ca}$ is the main constitutive element of teeth, presence of the trace elements relative to $\mathrm{Ca}$ can be determined.

Figure 8 show the identification of $\mathrm{Sr}$ in dentine and enamel of current and Middle Ages teeth. The shown spectra are typical of a collection of several pieces in each case. As it can be seen in the dentine of the latter there is a noticeable content of $\mathrm{Sr}$ compared with its own enamel and dentine and enamel of current teeth, which is low for both.

For the case of the analized neolitic teeth we found that the relation $\mathrm{Sr} / \mathrm{Ca}$ in dentine is almost twice that of current teeth. The fact that $\mathrm{Sr}$ appears in enamel may be related with dietary habits during the early period of growing. 

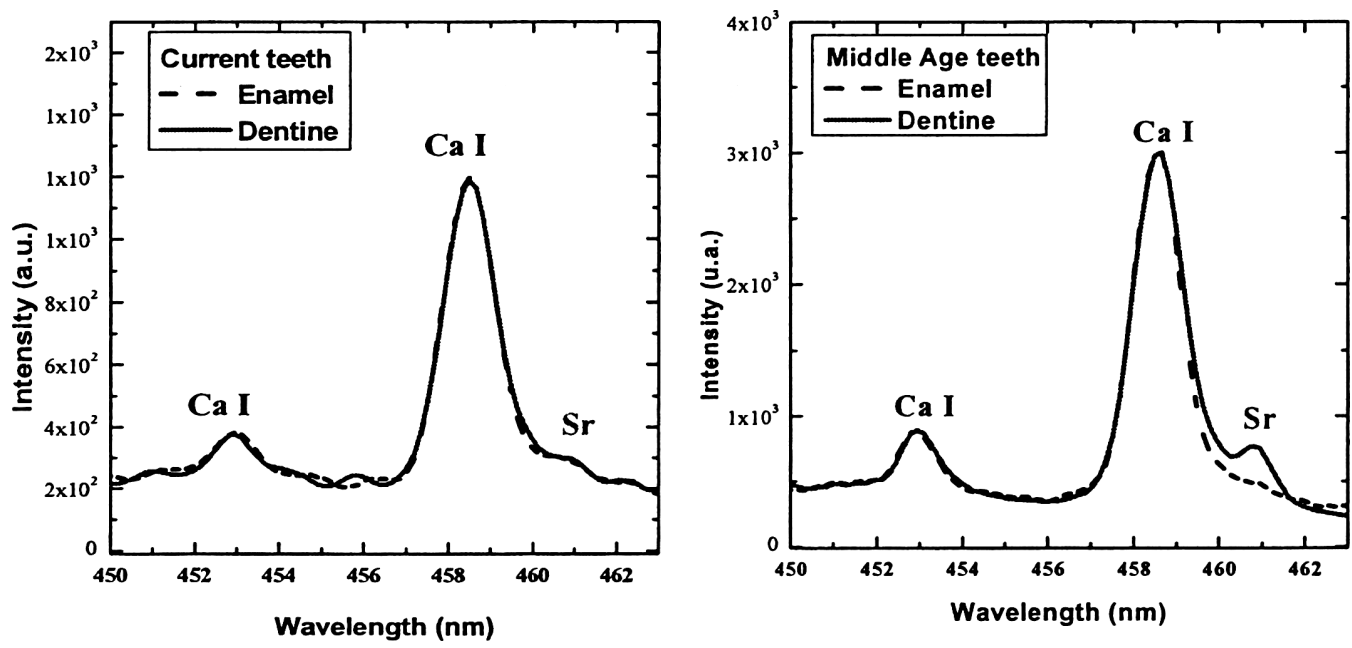

Figure 8. LIBS spectra of current and Middle Ages (south of France) teeth, showing the presence of Sr. Notice the typical $\mathrm{Ca} I$ line appearing in both teeth that can be used as reference.

Figure 9 illustrates the depth profiling ability of LIBS. Fig 9a is a photograph of part of an enamel neolitic tooth showing the ablation microdamage produced by increasing number of laser pulses. Figure $9 \mathrm{~b}$ shows the LIBS spectra obtained for selected number of laser shots $(1,2,20,100)$. Since each shot ablates a layer of material, the correspondig spectrum shows the element content of in-depth zones. Notice the change in the $\mathrm{Mg}$ line intensity as a function of depth. This result is important since it indicates that trace elements may be found non uniformly distributed in the piece. This information is usually lost when conventional methods that need special sample preparation are used.

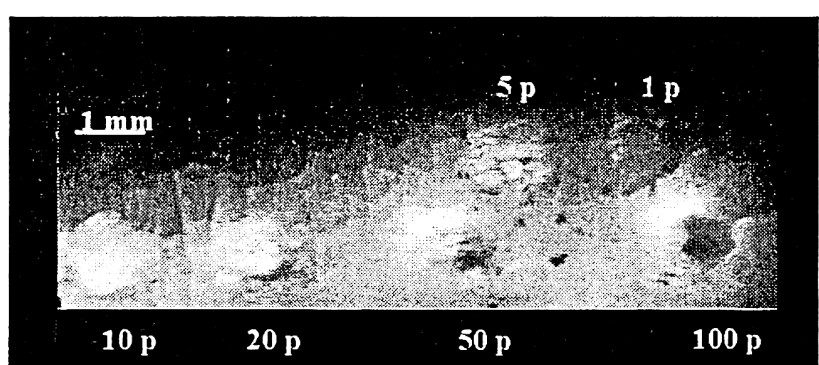

$\mathbf{a}$

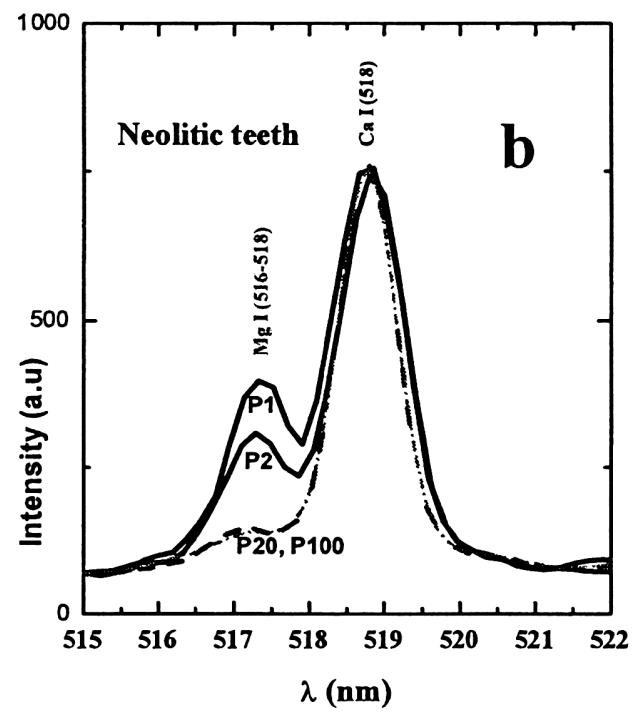

Figure 9. a) Photograph of part of an enamel neolitic tooth showing ablation microdamage as a function of the number of laser pulses (p). b) LIBS spectra corresponding to laser pulses number 1, 2, 20 and 100 impinging on the sample.

Figure 10 shows another application of LIBS, in this case to an archeological piece. The study was aimed to complement a more extensive analysis of a prehispanic bracelet of the Condorhuasi culture from the north of Argentina ${ }^{18}$. Concerning the delicateness of the piece, destructive analysis is prohibitive. In this case the LIBS 
technique allowed us to obtain the spectra of the main components of the bracelet by impinging the laser on the edge of a fixing hole, thus making almost imperceptible the microdestruction site. Besides, the use of a cross dispersion spectrograph and the synthesis of the $200-1100 \mathrm{~nm}$ spectrum obtained in a single record, allows obtaining the main components with few shots thus reducing the possibility of damage.

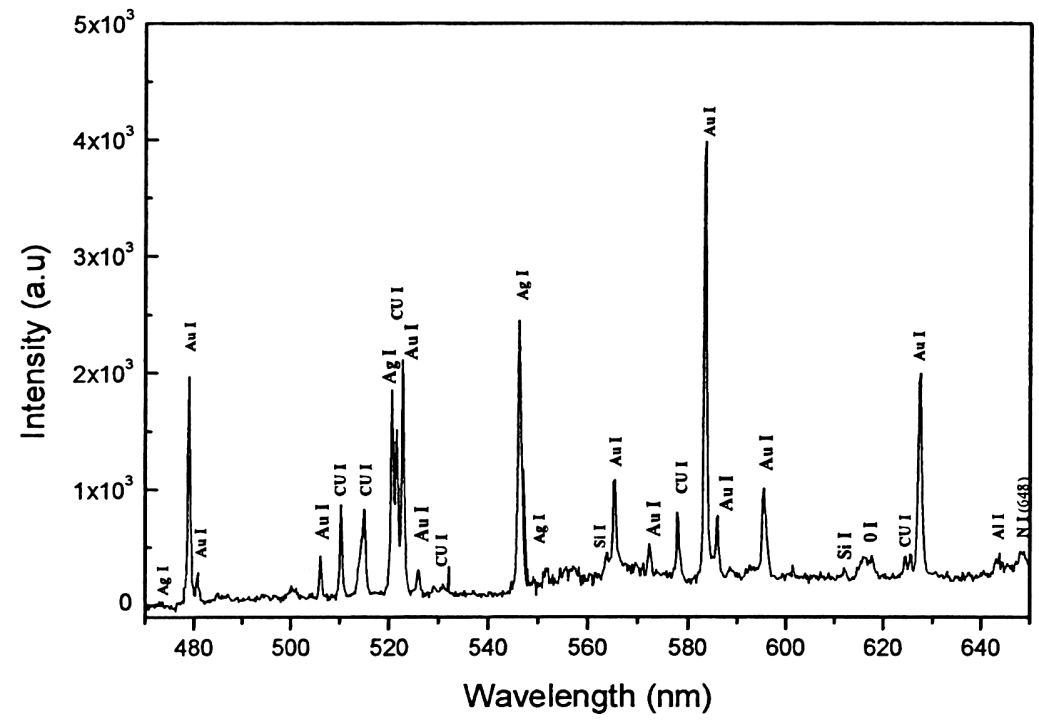

Fig 10. LIBS identification of the composition of a prehispanic bracelet of the Condorhuasi culture from the north of Argentina

\section{REFERENCES}

1-J.F. Asmus. Interdisciplinary Science Reviews 12, 171, 1987

2- K.G.Watkins, J.H. Lasrson, D.C.Emmony and W.M. Steen. Proc.Nato ASI on Laser Processing: Surface treatment and film Deposition. p.907, Kluwer Academic Publishers, 1995.

3- C.Fotakis. Optics and Photonics news. p.30, may 1995.

4- Journal of Cultural Heritage. Proc.LACONA III: Lasers in the conservation of Artworks. Vol 1. 2000. Elsevier

5- M.Cooper. Cleaning in conservation: an Introduction.Butterworth Heinemann, Oxford, 1998.

6- V.Zafiropulos in Laser Cleaning . Boris Luk ${ }^{r}$ yanchuk editor. World Scientific 2002.

7- G.M.Bilmes and O.E. Martínez, Patent AR990627, Argentina,1999.

8- G.M.Bilmes and O.E. Martínez, Patent 6.546.784- EEUU., 2003.

9- G.M.Bilmes, D.J. Orzi, O.E. Martínez, A. Lencina . Procc. Optical Measuremets Systems for industrial inspection

IV, SPIE International Symposium on Optical Metrology. 2005

10- D.Anglos Applied Spectroscopy, Vol.55, 6, p186A, 2001

11- J.M.Anzano, M.A. Villoria, I.B. Gormushkin; B.W. Smith, J.D. Winefordner Canadian Journal of Analytical Science and Spectroscopy, 47, 5, p134, 2002

12- D.J.O.Orzi and G. M. Bilmes, Applied Spectroscopy 58, pp.1475, 2004.

13- W. Kautek, S. Pentzien, P. Rudolph, J. Kruger, E. Konig. Appl. Surface Science, p746,1998.

14- J.Kolar, M. Strlic, S. Pentzien, W. Kautek. Appl. Phys A 71, 1, p87, 2000.

15- D.Muller-Hess LACLEPA Workshop 2000

16- M.Arronte, T. Flores, L. Ponce, G.M. Bilmes, III LACONA, 1999 and Patent 22804/2002, B08B 13/12. Cuba, 2002

17- G.M.Bilmes, D. Schinca F. Ramírez Rozzi, V Iberoamerican Meeting on Optics and VIII Latin American Meeting on Optics Lasers and their Applications Porlamar, Isla de Margarita, Venezuela 2004.

18-G.M.Bilmes, D.C. Schinca, M. Garavaglia, G.A.M. Alvarez . $4^{\text {th }}$ Iberoamerican Meeting on Optics and $7^{\text {th }}$ Latin American Meeting on Optics Lasers and their Applications. Tandil. Argentina.2001 